Supplementary material to:

\title{
Structural and Electronic Properties of Nanostructured HAlO
}

\author{
Yi Dong, ${ }^{1 *}$ Markus Burkhart,${ }^{2 \dagger}$ Michael Veith,${ }^{2}{ }^{\ddagger}$ and Michael Springborg ${ }^{1 \S}$ \\ ${ }^{1}$ Physical and Theoretical Chemistry, ${ }^{2}$ Inorganic Chemistry, \\ University of Saarland, Postfach 1511 50, D - 66041 Saarbrücken, Germany
}

(Dated: September 10, 2005)

\footnotetext{
* e-mail: y.dong@mx.uni-saarland.de

$\dagger$ e-mail: m.burkhart@mx.uni-saarland.de

$\ddagger$ e-mail: veith@mx.uni-saarland.de

$\S$ e-mail: m.springborg@mx.uni-saarland.de
} 


\section{DFT CALCULATIONS}

In the parameter-free density-functional calculations we constructed various structures that subsequently were allowed to relax to their closest total-energy minima. The structures as well as the notation we have used in classifying them are all shown in Fig. 1. The results of the density-functional calculations are given in Table I. 
TABLE I: Calculated energy and interatomic distances for $[\mathrm{HAlO}]_{n}(n=1,2,3,4,5,6)$. The energy has been corrected for zero-point energy. The distances correspond to mean values where applicable. Indices $t$ and $b$ describe terminal and bridging atoms. Symmetry is given as calculation/final point group.

\begin{tabular}{|c|c|c|c|c|c|c|c|c|c|c|}
\hline Descriptor & $E_{\text {corr }}[$ a.u. $]$ & $\begin{array}{l}\text { Point- } \\
\text { group }\end{array}$ & $r\left(\mathrm{Al}-\mathrm{H}_{t}\right)$ & $r\left(\mathrm{Al}-\mathrm{H}_{b}\right)$ & $r\left(\mathrm{Al}-\mathrm{O}_{t}\right)$ & $\begin{array}{l}\text { Distances } \\
r\left(\mathrm{Al}^{-\mathrm{O}_{b}}\right)\end{array}$ & $\begin{array}{l}[\AA]] \\
r(\mathrm{Al}-\mathrm{Al})\end{array}$ & $r(\mathrm{O}-\mathrm{O})$ & $r(\mathrm{H}-\mathrm{H})$ & $r(\mathrm{O}-\mathrm{H})$ \\
\hline \multicolumn{11}{|l|}{$[\mathrm{HAlO}]_{1}$} \\
\hline aloh & -318.16322 & $\mathrm{C}_{6 v} / \mathrm{C}_{\infty v}$ & & & & 1.6996 & & & & 0.9677 \\
\hline halo & -318.11333 & $\mathrm{C}_{6 v} / \mathrm{C}_{\infty v}$ & 1.5871 & & 1.6221 & & & & & \\
\hline \multicolumn{11}{|l|}{$[\mathrm{HAlO}]_{2}$} \\
\hline lin-mu-o & -636.42522 & $\mathrm{D}_{2 h}$ & 1.5849 & & & 1.7697 & 2.4256 & 2.5776 & 5.5953 & \\
\hline lin-mu-oh & -636.37530 & $\mathrm{D}_{2 h}$ & & & & 1.9306 & 3.0680 & 2.3442 & 4.3011 & 0.9784 \\
\hline lin-mu-oo & -636.35228 & $\mathrm{C}_{2 v}$ & 1.5952 & & 1.6214 & 1.7075 & 3.4148 & 3.3104 & 2.8383 & \\
\hline al2oh2 & -636.33029 & $\mathrm{C}_{2}$ & & & & 1.7054 & 3.0782 & 5.5354 & 6.8824 & 0.9694 \\
\hline lin-mu-h & -636.23347 & $\mathrm{D}_{2 h}$ & & 1.7588 & 1.6281 & & 2.5993 & 5.8554 & 2.3702 & \\
\hline \multicolumn{11}{|l|}{$[\mathrm{HAlO}]_{3}$} \\
\hline ring-mu-o & -954.72562 & $\mathrm{D}_{3 d}$ & 1.5851 & & & 1.7324 & 3.0751 & 2.9199 & 5.8206 & \\
\hline plane-mu-o & -954.67121 & $\mathrm{C}_{s}$ & 1.5973 & & & 1.8134 & 2.9355 & 2.8780 & 4.7145 & \\
\hline lin-mu-oo & -954.67032 & $\mathrm{C}_{1}$ & 1.5933 & & & 1.7466 & 3.8840 & 2.9748 & 6.4606 & \\
\hline trig-mu-h & -954.58814 & $\mathrm{C}_{2 v}$ & 1.5751 & 1.7739 & 1.6276 & 1.9016 & 2.5652 & 3.0237 & 3.7617 & \\
\hline lin-2mu-ho & -954.55361 & $\mathrm{C}_{1}$ & 1.5806 & 1.7576 & 1.6267 & 1.7683 & 3.3397 & 4.5752 & 4.4134 & \\
\hline al3oh3 & -954.51875 & $\mathrm{C}_{1}$ & & & & 1.7286 & 2.7300 & 5.3566 & 6.5476 & 0.9755 \\
\hline ring-mu-h & -954.36277 & $\mathrm{D}_{3 d}$ & & 1.7363 & 1.6275 & & 3.3488 & 6.1673 & 2.4700 & \\
\hline \multicolumn{11}{|l|}{$[\mathrm{HAlO}]_{4}$} \\
\hline cub-mu-o & -1273.05037 & $\mathrm{~T}_{d}$ & 1.5806 & & & 1.8749 & 2.6772 & 2.6252 & 5.2583 & \\
\hline ring-mu-o & -1272.98709 & $\mathrm{D}_{4 h}$ & 1.5859 & & & 1.7178 & 3.7769 & 3.3858 & 6.3294 & \\
\hline
\end{tabular}


TABLE I: (continued)

\begin{tabular}{|c|c|c|c|c|c|c|c|c|c|c|}
\hline \multirow[b]{2}{*}{ Descriptor } & \multirow[b]{2}{*}{$E_{\text {corr }}[$ a.u. $]$} & \multirow{2}{*}{$\begin{array}{l}\text { Point- } \\
\text { group }\end{array}$} & \multicolumn{8}{|c|}{ Distances $[\AA]$} \\
\hline & & & $r\left(\mathrm{Al}-\mathrm{H}_{t}\right)$ & $r\left(\mathrm{Al}-\mathrm{H}_{b}\right)$ & $r\left(\mathrm{Al}-\mathrm{O}_{t}\right)$ & $r\left(\mathrm{Al}-\mathrm{O}_{b}\right)$ & $r(\mathrm{Al}-\mathrm{Al})$ & $r(\mathrm{O}-\mathrm{O})$ & $r(\mathrm{H}-\mathrm{H})$ & $r(\mathrm{O}-\mathrm{H})$ \\
\hline lin-mu-ooo & -1272.91490 & $\mathrm{C}_{1}$ & 1.5981 & & & 1.7348 & 5.0132 & 3.5084 & 8.2092 & \\
\hline lin-mu-oho & -1272.87182 & $\mathrm{C}_{1}$ & 1.5828 & 1.7531 & & 1.7633 & 4.1403 & 4.3977 & 5.7720 & \\
\hline al4oh4 & -1272.76716 & $\mathrm{~S}_{4}$ & & & & 1.7205 & 2.6366 & 5.4417 & 6.6758 & 0.9730 \\
\hline ring-mu-ho & -1272.72861 & $\mathrm{C}_{2 h}$ & 1.5838 & 1.7488 & 1.6271 & 1.7789 & 3.8537 & 4.8416 & 4.7634 & \\
\hline ring-mu-h & -1272.48132 & $\mathrm{C}_{1}$ & & 1.7370 & 1.6270 & & 3.9534 & 6.5720 & 2.8265 & \\
\hline cub-mu-h & -1272.47012 & $\mathrm{~T}_{d}$ & & 1.8777 & 1.6249 & & 2.9478 & 5.6012 & 2.2782 & \\
\hline$[\mathrm{HAlO}]_{6}$ & & & & & & & & & & \\
\hline ring2x6-mu-o & -1909.65698 & $\mathrm{D}_{3 d}$ & 1.5814 & & & 1.8603 & 3.2486 & 3.0481 & 5.6654 & \\
\hline cub+6-ha' & -1909.58188 & $\mathrm{C}_{1}$ & 1.5825 & 1.7881 & & 1.8575 & 3.4577 & 3.1810 & 5.6753 & \\
\hline cub+6-hi & -1909.57340 & $\mathrm{C}_{s}$ & 1.5849 & & & 1.8290 & 3.6694 & 3.0216 & 5.3431 & \\
\hline cub+6-ha & -1909.56678 & $\mathrm{C}_{s}$ & 1.5894 & & & 1.8548 & 3.6021 & 3.2393 & 5.3452 & \\
\hline cub+2x2z-hi & -1909.56360 & $\mathrm{C}_{1}$ & 1.5886 & & & 1.8584 & 3.2685 & 3.1870 & 5.6223 & \\
\hline cub $+2 \times 2 e-h i$ & -1909.56193 & $\mathrm{C}_{2 v}$ & 1.5827 & & & 1.8381 & 3.5053 & 3.3162 & 5.7434 & \\
\hline $\mathrm{ada}+2 \mathrm{x} 1 \mathrm{e}$ & -1909.54773 & $\mathrm{C}_{1}$ & 1.5886 & 1.7933 & & 1.8362 & 3.5065 & 3.1243 & 5.4868 & \\
\hline ring2x6-4 & -1909.54571 & $\mathrm{C}_{i}$ & 1.5896 & & & 1.7784 & 4.2039 & 4.0339 & 6.2067 & \\
\hline cub+6-ha" & -1909.53883 & $\mathrm{C}_{s}$ & 1.5892 & & & 1.8485 & 3.6943 & 3.4691 & 5.9060 & \\
\hline lin-mu-hoooh & -1909.53550 & $\mathrm{C}_{1} / \mathrm{C}_{2}$ & 1.5842 & 1.8061 & & 1.8335 & 3.9707 & 3.5569 & 6.2156 & \\
\hline plane-mu-o & -1909.53087 & $\mathrm{C}_{i}$ & 1.5874 & & & 1.8384 & 4.1983 & 4.1216 & 5.9853 & \\
\hline cub+2x2z-ha & -1909.52826 & $\mathrm{C}_{2}$ & 1.5937 & & & 1.8899 & 3.4395 & 3.2350 & 5.6831 & \\
\hline cub+2x2e-ha & -1909.52782 & $\mathrm{C}_{2 v}$ & 1.5933 & & & 1.8876 & 3.5428 & 3.3232 & 5.4311 & \\
\hline ring2x6-6 & -1909.52572 & $\mathrm{C}_{s}$ & 1.5868 & & & 1.8018 & 3.7007 & 3.3834 & 5.9281 & \\
\hline cub2x-ha & -1909.51807 & $\mathrm{C}_{1}$ & 1.5934 & & & 1.8871 & 3.3077 & 3.0947 & 5.5340 & \\
\hline $\mathrm{ada}+2 \mathrm{x} 2 \mathrm{e}-\mathrm{hi}$ & -1909.51031 & $\mathrm{C}_{1} / \mathrm{C}_{2}$ & 1.5849 & 1.7640 & & 1.7874 & 3.8175 & 3.7871 & 5.4199 & \\
\hline $\mathrm{ada}+2 \mathrm{x} 2 \mathrm{z}-\mathrm{hi}$ & -1909.50526 & $\mathrm{C}_{1} / \mathrm{C}_{s}$ & 1.5852 & 1.7868 & & 1.7888 & 3.7455 & 3.7812 & 5.2133 & \\
\hline ada $+2 \times 1$ e-ha & -1909.46485 & $\mathrm{C}_{1}$ & 1.5975 & 1.7842 & & 1.7905 & 3.7780 & 3.1773 & 5.9154 & \\
\hline
\end{tabular}


TABLE I: (continued)

\begin{tabular}{|l|c|c|c|c|c|c|c|c|c|c|}
\hline \hline & & Point- & \multicolumn{7}{|c|}{ Distances $[\AA]$} \\
Descriptor & $E_{\text {corr }}$ [a.u. $]$ & group & $r\left(\mathrm{Al}-\mathrm{H}_{t}\right)$ & $r\left(\mathrm{Al}_{\mathrm{H}}\right)$ & $r\left(\mathrm{Al}_{t}\right)$ & $r\left(\mathrm{Al}-\mathrm{O}_{b}\right)$ & $r(\mathrm{Al}-\mathrm{Al})$ & $r(\mathrm{O}-\mathrm{O})$ & $r(\mathrm{H}-\mathrm{H})$ & $r(\mathrm{O}-\mathrm{H})$ \\
\hline ada+2x1z-ha & -1909.46166 & $\mathrm{C}_{1}$ & 1.5968 & 1.7800 & & 1.7926 & 3.7098 & 3.1794 & 5.3807 & \\
ada+2x1z-hi1 & -1909.45455 & $\mathrm{C}_{1}$ & 1.5908 & & & 1.8502 & 3.9051 & 3.1589 & 5.9548 & \\
ring6+3x2-ha & -1909.44613 & $\mathrm{C}_{1}$ & 1.6029 & & & 1.7605 & 4.2643 & 3.9692 & 6.6531 & \\
ring2x6-mu-ho & -1909.40234 & $\mathrm{C}_{3}$ & 1.5921 & & & 1.8918 & 3.5211 & 3.0666 & 4.2533 & 0.9809 \\
lin-mu-oohoo & -1909.36173 & $\mathrm{D}_{2 h}$ & 1.5977 & 1.7516 & & 1.7455 & 6.3873 & 5.4487 & 9.0537 & \\
ring2x6-mu-h & -1909.33280 & $\mathrm{C}_{1}$ & 1.5841 & 1.8174 & & 1.7394 & 3.8189 & 4.4422 & 4.0385 & 0.9746 \\
lin-mu-ohoho & -1909.31745 & $\mathrm{C}_{1}$ & 1.5826 & 1.7523 & & 1.7676 & 5.8083 & 6.0474 & 6.9560 & \\
plane-mu-h & -1909.26593 & $\mathrm{C}_{i}$ & 1.5827 & 1.7633 & 1.6205 & 1.7081 & 5.0841 & 6.5811 & 4.0594 & \\
oct-al6oh6 & -1909.13194 & $\mathrm{C}_{1}$ & & & & 1.7321 & 3.0201 & 5.5199 & 6.4369 & 0.9766 \\
lin-mu-hohoh & -1909.12557 & $\mathrm{D}_{2 h}$ & & 1.7544 & 1.6262 & 1.7663 & 5.8389 & 7.0977 & 5.9834 & \\
\hline \hline
\end{tabular}




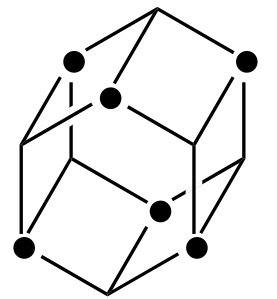

ring $2 \times 6$

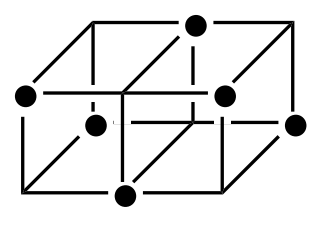

cub2x

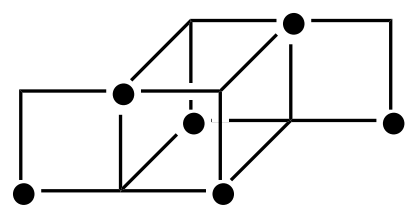

$c u b+2 \times 2 e$

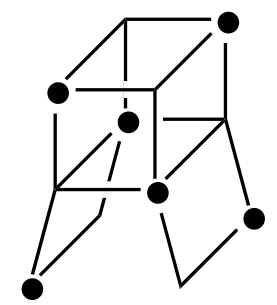

$c u b+2 \times 2 z$

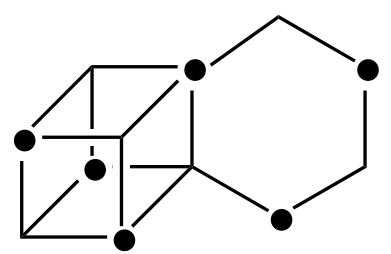

cub+6

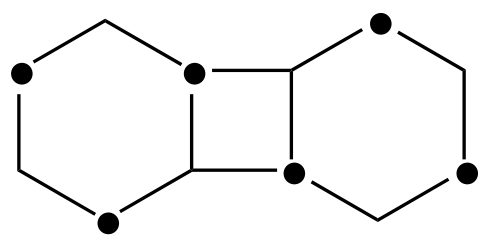

ring $2 \times 6-4$

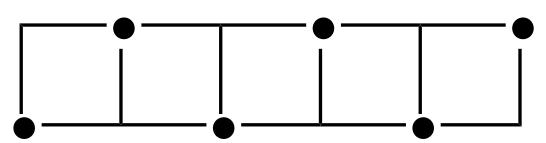

plane

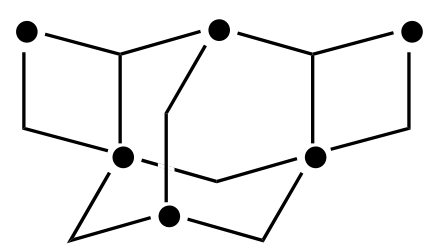

$\operatorname{ada}+2 \times 2 z$

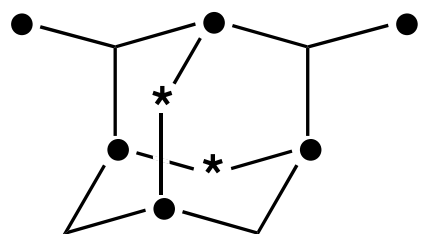

$\operatorname{ada}+2 \mathrm{x} 1 \mathrm{z}$

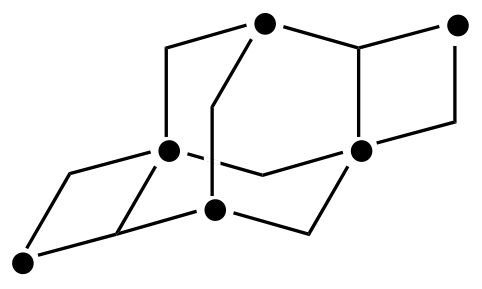

$\operatorname{ada}+2 \times 2 e$

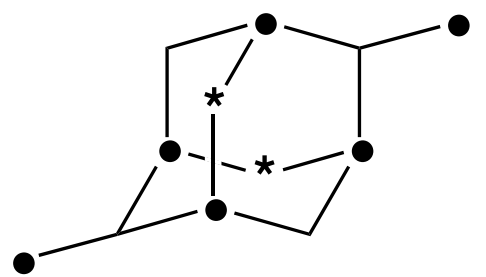

$\operatorname{ada}+2 \times 1 e$

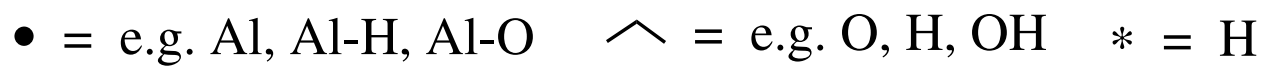

FIG. 1: The structures and their notation that were considered in the parameter-free densityfunctional calculations. 